\title{
Catégories phonologiques et représentation des mots dans le développement lexical de l'enfant bilingue
}

Laura Bosch, Marta Ramon-Casas et Núria Sebastián Gallés

\section{OpenEdition}

\section{Journals}

Édition électronique

URL : http://journals.openedition.org/rlv/1429

DOI : $10.4000 /$ rlv. 1429

ISSN : 1958-9239

Éditeur

Presses universitaires de Vincennes

Édition imprimée

Date de publication : 1 mai 2006

Pagination : 53-76

ISBN : 978-2-84292-208-5

ISSN : 0986-6124

\section{Référence électronique}

Laura Bosch, Marta Ramon-Casas et Núria Sebastián Gallés, « Catégories phonologiques et représentation des mots dans le développement lexical de l'enfant bilingue », Recherches linguistiques de Vincennes [En ligne], 35 | 2006, mis en ligne le 22 décembre 2006, consulté le 19 avril 2019. URL : http://journals.openedition.org/rlv/1429; DOI : 10.4000/rlv.1429 
Recherches linguistiques de Vincennes 35 - 2006 - p. 53-76

\title{
Laura BOSCH, Marta RAMON-CASAS \& Núria SEBASTIÁN-GALLÉS Université de Barcelone, Espagne
}

\author{
CATÉGORIES PHONOLOGIQUES ET REPRÉSENTATION \\ DES MOTS DANS LE DÉVELOPPEMENT LEXICAL \\ DE L'ENFANT BILINGUE ${ }^{1}$
}

\begin{abstract}
RÉSUMÉ
Cet article analyse l'émergence des catégories contrastives dans le développement phonologique initial bilingue. On présente différents travaux qui, au cours de la première année de vie, ont évalué les capacités précoces de différentiation entre les langues de l'entourage, la discrimination des sons de la parole à l'étape pré-lexicale, la familiarité avec les patrons phonotactiques qui caractérisent la langue et la continuité entre toutes ces habilités précoces et leur utilisation dans la reconnaissance des premiers mots. La comparaison entre deux situations d'acquisition différentes, monolingue et bilingue, permet de constater le rôle de l'expérience perceptive dans l'organisation phonologique initiale. Les travaux analysés montrent que la capacité pour distinguer entre les deux langues de l'entourage, même quand elles ont des propriétés rythmiques très semblables, est possible dès l'age de 4 mois. Le processus de réorganisation perceptive, qui a lieu vers la fin de la première année de vie et qui montre le début de l'organisation contrastive de sons, suit un développement différent chez le bilingue en montrant un petit « retard » quand on le compare avec le monolingue. La connaissance des patrons phonotactiques suggère une prédominance linguistique chez le bilingue qui favorise la langue qui est plus présente dans l'entourage. Finalement, les travaux qui ont analysé la reconnaissance des mots familiers et leur format de représentation dans la deuxième année de vie montrent aussi un décalage temporel en ce qui concerne la représentation d'un contraste vocalique qui appartient à une des deux langues familières. On constate la spécificité du développement phonologique initial chez le bilingue qui doit faire des adaptations précises pour faire face à la nature plus riche et complexe de son input linguistique.
\end{abstract}

\section{MoTS-CLÉS}

Acquisition bilingue, perception de la parole, catégories contrastives, reconnaissance de mots, phonologie initiale. 


\section{Introduction}

Au cours de l'acquisition du langage chez le bilingue, la découverte de l'existence de deux systèmes de sons différents dans l'environnement est considérée comme un accomplissement nécessaire au développement phonologique. Ainsi, la différenciation précoce entre les deux langues de l'environnement est une condition préalable à un processus d'acquisition qui permet le développement harmonieux des deux systèmes phonologiques. Les capacités de discrimination des jeunes bébés ont été évaluées chez des bébés monolingues (Mehler et al., 1988 ; Bahrick \& Pickens, 1988 ; Moon et al., 1993 ; Mehler \& Christophe, 1994 ; Christophe \& Morton, 1998 ; DehaeneLambertz \& Houston, 1998 ; Nazzi et al., 1998 ; Nazzi et al., 2000 ; Bosch et al., 2001). Les résultats ont montré que les nouveau-nés peuvent différencier très précocement certaines langues mais pas d'autres. On a montré, par exemple, que les nouveau-nés peuvent distinguer entre l'espagnol et l'anglais (Moon et al., 1993) et entre l'anglais et le japonais, mais pas entre le hollandais et l'anglais ou entre l'espagnol et l'italien par exemple (Nazzi et al., 1998). Ceci laisse donc penser que la ressemblance structurelle entre les inputs présentés va diminuer la possibilité pour les enfants de discriminer deux langues. Plus généralement, l'hypothèse actuellement retenue suggère que les nouveau-nés ne peuvent distinguer des langues que si elles diffèrent par des aspects fondamentaux de leur structure rythmique ou prosodique (Abercrombie, 1967), mais qu'ils ne peuvent pas les discriminer quand elles appartiennent à la même catégorie rythmique (Mehler et al., 1988; Nazzi et al., 1998). Cette capacité n'est pas spécifique aux êtres humains, puisque elle peut être observée chez d'autres mammifères, comme les singes de tamarin (Ramus et al., 2000) et les rats (Toro et al., 2005). Indépendamment de l'origine phylogénétique de cette capacité, la familiarité avec la structure prosodique de la langue d'exposition a été considérée par différents chercheurs comme le noyau de l'hypothèse du BOOTSTRAPPING prosodique pour l'acquisition lexicale et syntaxique (Guasti et al., 2001). On entend de manière générale par bootstrapping l'utilisation de connaissances déjà acquises pour faciliter l'acquisition de nouvelles aptitudes, pour d'autres composantes de la grammaire. Plus concrètement, le bootstrapping prosodique ou phonologique propose que l'enfant, au cours de l'acquisition du langage, se serve des informations prosodiques pour segmenter la parole continue en unités plus petites, à partir desquelles il va extraire des informations rudimentaires lui permettant d'élaborer de nouvelles hypothèses syntaxiques et lexicales. Dans les environnements bilingues, ce phénomène de bootstrapping prosodique peut être particulièrement utile pour l'acquisition des contrastes phonologiques, puisque la prosodie comporte des informations permettant la discrimination 
entre les langues après une courte période d'exposition (probablement quelques mois seulement). Si l'hypothèse de bootstrapping prosodique est juste, l'information prosodique pourrait faciliter la découverte des régularités propre à chacune des deux langues et les bébés pourraient utiliser cette information pour commencer à construire les deux systèmes phonologiques séparément, bien avant qu'ils n'atteignent l'étape lexicale dans leur développement linguistique.

Dans cet article, nous présentons les résultats de travaux sur l'émergence des catégories phonologiques contrastives dans le développement phonologique initial bilingue. Nous partirons des études qui montrent la capacité précoce de différentiation entre les langues parlées dans l'entourage linguistique de l'enfant. Nous examinerons la capacité des bébés bilingues à discriminer les sons de la parole dans deux langues différentes durant l'étape prélexicale. Ceci nous permettra de mettre en évidence une continuité entre les capacités perceptives de la première année de vie et leur utilisation par les enfants dans la reconnaissance des premiers mots. Enfin, nous comparerons deux situations d'acquisition différentes (les développements monolingue et bilingue) pour analyser plus précisément le rôle de l'expérience perceptive dans l'organisation de la phonologie.

\section{La discrimination des langues par le bébé en environnement bilingue}

Combien de temps faut-il pour qu'un enfant bilingue puisse séparer deux systèmes linguistiques auxquels il est exposé simultanément? Des études faites dans notre laboratoire avec des bébés exposés dès la naissance au catalan et à l'espagnol (deux langues romanes appartenant au même groupe rythmique, voir Ramus et al., 1999) ont montré que dès l'âge de 4 mois et demi, les bébés bilingues peuvent distinguer les deux langues. Dans cette recherche, les bébés ont été familiarisés avec six phrases différentes dans leur langue maternelle (définie comme la langue parlée par leur mère d'une manière prédominante, que ce soit le catalan ou l'espagnol, deux groupes différents de bébés bilingues ont donc été testés dans l'étude). Après deux minutes de familiarisation, huit phrases, inconnues d'eux leur ont été présentées, la moitié de celles-ci étaient produites dans la langue de la phase de familiarisation et l'autre moitié dans l'autre langue. La locutrice était la même. Dans les deux cas, cette femme a adopté un style MOTHERESE qui se caractérisait par un patron intonatif plus exagéré et un débit d'émission plus lent que celui qu'on utilise quand on s'adresse à un adulte. La méthodologie utilisée était le paradigme de FAMILIARISATION-PRÉFÉRENCE, qui permet de mesurer le temps d'attention par la fixation visuelle d'images sur des moni- 
teurs latéraux, pendant que les stimuli auditifs (les phrases) sont présentés. L'expérimentateur enregistre le regard de l'enfant : à chaque essai, l'enfant entend les phrases aussi longtemps qu'il fixe l'attention sur un des moniteurs ; par contre, l'essai est interrompu quand il cesse de fixer son attention pendant plus de deux secondes. Quand l'enfant détourne ainsi son attention pendant plus de deux secondes consécutives un nouvel essai commence et on continue de cette façon jusqu'à ce que l'enfant ait complété les huit essais de la phase test. Les deux groupes de bébés ont montré des différences significatives de temps d'attention entre les essais même-langue et les essais autrelangue. Des temps d'attention plus longs sont observés pour les phrases dans la langue considérée comme n'étant pas la langue maternelle. Ceci s'interprète comme un indice de discrimination (Bosch \& Sebastián-Gallés, 2001a). Des résultats similaires ont été obtenus avec deux groupes indépendants de bébés qui procédaient d'environnements monolingues (catalans et espagnols), ce qui nous indique que, contrairement à ce qui avait été montré par Mehler et al. (1998) et Nazzi et al. (1998), ces deux langues appartenant à la même famille romane peuvent être différenciées précocement dans le développement, indépendamment du niveau et de la qualité d'exposition reçue.

Par ailleurs, ces résultats montrent que, de façon importante, l'exposition bilingue simultanée n'a pas créé de problèmes spécifiques dans le processus de différenciation de cette paire de langues, pour lesquelles il était prédit que la différentiation ne pouvait être observable qu'à une étape plus tardive (Mehler et al., 1996).

Une autre étude, réalisée avec des nord-américains monolingues exposés exclusivement à l'anglais, a montré qu'à l'âge de 5 mois ces bébés sont déjà capables de faire quelques discriminations entre langues d'un même groupe rythmique, mais à condition que ces langues appartiennent à la même catégorie rythmique que la langue maternelle et que l'une d'entre elles soit cette langue (ou une langue très proche, du même groupe rythmique). Par exemple, Jusczyk et ses collaborateurs ont observé que des bébés monolingues américains de 5 mois (pas avant) pouvaient discriminer entre l'anglais britannique et le hollandais (les deux de type accentuel) mais également entre deux dialectes de l'anglais (l'anglais britannique et l'anglais américain). Par contre, ils ne pouvaient pas distinguer entre le hollandais et l'allemand, bien qu'il s'agisse de deux langues de même type accentuel, car aucune d'entre elles n'était la langue maternelle (Nazzi et al., 2000). Sur la base de ces résultats, les auteurs ont émis l'hypothèse que la capacité à faire des discriminations intra-catégorie émergerait comme une conséquence de l'apprentissage graduel chez le bébé des traits rythmiques spécifiques à la langue maternelle. Cet approfondissement de la connaissance du patron rythmique natif permettrait d'établir la différentiation entre des langues de la même catégorie : la 
langue maternelle servant de référence à partir de laquelle le bébé établirait ses comparaisons.

Une hypothèse alternative peut néanmoins être aussi envisagée qui relativise l'importance du bootstrapping prosodique. Celle-ci envisage que cette capacité de différenciation puisse être conditionnée aussi par la fréquence et la distribution des sons vocaliques dans les langues à discriminer. Dans une recherche basée sur des langues de type syllabique, les bébés des familles monolingues espagnoles et monolingues catalanes ont été évalués pour leur capacité à discriminer à l'âge de 4 mois et demi des inputs en catalan, espagnol et italien (Bosch \& Sebastián-Gallés, 2000). Les bébés monolingues distinguaient aisément l'espagnol du catalan ainsi que l'italien du catalan, alors qu'ils ne pouvaient pas distinguer l'espagnol de l'italien. Ces résultats ne peuvent pas être attribués à des différences typologiques majeures puisque ces trois langues sont des langues romanes, montrant, de plus, des différences prosodiques minimales: les données des études avec des participants adultes sur ces mêmes matériels filtrés à $400 \mathrm{~Hz}$ ont permis de le montrer (voir Bosch \& Sebastián-Gallés, 2000). Au contraire, les résultats sont attribués aux propriétés distributionnelles spécifiques des voyelles dans ces trois langues : l'italien et l'espagnol partagent une distribution plus similaire de sons vocaliques (les voyelles /a/, /e/, /i/ et /o/ ont une fréquence assez comparable dans la parole continue dans ces deux langues), qui diffère de celle du catalan, où les voyelles centrales /a/ et schwa comptent pour plus de la moitié du nombre total de voyelles présentes dans la parole continue.

De même, les enfants monolingues de 4 mois et demi sont capables de différencier deux dialectes du catalan dont la distinction est fondée sur le répertoire et la distribution des sons vocaliques (Bosch \& SebastianGallés, 2004). L'absence de réduction vocalique dans le dialecte occidental détermine une notable différence entre ces deux dialectes du catalan, qui se manifeste exclusivement par le nombre de sons vocaliques dans ces deux dialectes ainsi que par leur distribution dans la parole continue. Le résultat est que le catalan occidental possède un système de voyelles et une distribution de celles-ci beaucoup plus proche de l'espagnol que du catalan centraloriental. Ceci pourrait expliquer la facilité que manifestent les enfants pour discriminer ces langues, alors que du point de vue phonotactique, il n'y a pas de différences qui pourrait les aider.

Ces résultats montrent combien l'information distributionnelle, segmentale semble jouer un rôle dans la capacité des bébés à discriminer leur langue maternelle d'une autre langue, alors qu'on avait plutôt considéré qu'ils s'appuyaient prioritairement sur des différences prosodiques et plus spécialement sur des différences rythmiques. D’autres résultats sont nécessaires pour confirmer ou réfuter cette explication alternative et clarifier les 
limites de sa généralité, mais ces premières données invitent à considérer que, juste avant l'âge de 6 mois, les bébés des environnements monolingues ou bilingues peuvent commencer à distinguer des langues qui sont rythmiquement très proches sur la base de la seule information segmentale, vocalique.

Ces résultats ont, en outre, une autre portée. Ils suggèrent que l'extraction des propriétés distributionnelles relatives à des éléments segmentaux spécifiques de la langue cible, les voyelles par exemple, est précoce et que, dès 6 mois, à partir des langues disponibles dans leur environnement, les bébés pourraient être capables de tirer des généralisations segmentales. Bien que cette hypothèse s'écarte du modèle TIGRE proposé par Mehler et al. (1996), qui base les capacités de discrimination des langues exclusivement sur l'information prosodique, on pourrait aussi considérer que l'importance attribuée aux voyelles dans ce modèle n'élimine pas la possibilité que deux types d'information puissent éventuellement être extraits et exploités : information rythmique ET information distributionnelle, relative aux segments vocaliques de la langue en question. Le travail de Ramus et al. (1999), - qui considère que la perception précoce et la découverte des patterns rythmiques d'une langue s'appuient sur la perception des voyelles, reconnues comme saillantes alors que les consonnes sont perçues et traitées comme des intervalles de bruits entre deux voyelles -, permet aussi de faire l'hypothèse que la distribution des sons vocaliques dans la parole continue joue un rôle dans l'acquisition précoce.

En ce qui concerne les enfants bilingues, des études menées dans notre laboratoire ont montré qu'à 4 mois et demi et à 6 mois, les bébés bilingues discriminent entre leur langue maternelle, soit le catalan ou soit l'espagnol, et l'anglais, qui appartient à une catégorie rythmique différente. Cependant, leur patron de réponses est clairement différent du patron obtenu avec les bébés monolingues pour ce même contraste (Bosch \& SebastiánGallés, 1997 ; Bosch \& Sebastián-Gallés, 2001b). Dans le paradigme expérimental utilisé dans ces études, qui se base sur la mesure de la latence d'orientation vers des phrases dans une langue familière (maternelle) versus des phrases dans une langue inconnue pour l'enfant, on s'attend à une orientation plus rapide pour les phrases dans la langue familière. Le patron des bilingues, par contre, montre des latences d'orientation significativement différentes entre les phrases de la langue maternelle et les phrases en anglais, mais la latence dans ce cas est plus lente pour le matériel familier que pour le matériel non familier. Même si on n'a pas encore une explication satisfaisante pour ce patron de réponses spécifique du bilingue, il est un clair indice qu'il y a une adaptation précoce des mécanismes de traitement de la parole pour un input linguistique bilingue, plus riche et varié que celui de l'enfant 
monolingue. De plus, on observe chez le bilingue la possibilité de différencier entre l'italien (langue syllabique, mais non familière) et la langue maternelle, soit l'espagnol ou le catalan. Autrement dit, l'enfant d'un entourage bilingue serait capable de faire une distinction précoce entre des langues de la même catégorie prosodique, ce qui n'a pas pu être observé chez l'enfant monolingue de 4 mois et demi, pour le contraste entre l'espagnol et l'italien (Bosch \& Sebastián-Gallés, 1997).

Les résultats semblent donc suggérer que des facteurs qui aident le bébé à atteindre une première différenciation entre les langues lors de l'exposition bilingue sont :

(a) l'attention aux caractéristiques prosodiques spécifiques de chacune des langues, quand elles existent ;

(b) la connaissance des propriétés distributionnelles dans la parole continue des éléments syllabiques ou segmentaux (les voyelles, par exemple).

Cela étant dit, les mécanismes spécifiques impliqués vont varier en fonction des contrastes opposant les langues et on peut même faire l'hypothèse que l'on va pouvoir observer des différences assez importantes en comparant les aptitudes précoces des bilingues qui sont exposés à des langues de la même classe rythmique versus ceux qui sont exposés à deux langues prosodiquement très éloignées.

\section{La construction des catégories phonétiques de la langue maternelle}

La recherche sur une population monolingue a déjà montré que, dès l'âge de six mois, les bébés manifestent un comportement qui traduit une certaine réorganisation perceptive, privilégiant les catégories de sons de la langue maternelle. Plusieurs travaux ont étudié les changements qui ont lieu au cours de la première année de vie dans la perception des segments consonantiques et leur relation avec les catégories contrastives natives. Le travail pionnier de Werker \& Tees (1984) a montré un déclin de la sensibilité des bébés aux contrastes phonétiques non natifs pendant la première année de vie, appelé couramment «surdité phonologique ». Des bébés canadiens de familles anglophones ont été testés sur trois contrastes différents, un contraste de la langue maternelle $(/ \mathrm{da} /-/ \mathrm{ta} /)$, et deux contrastes correspondant à des langues non familières (un contraste de l'occlusive rétroflexe $v s$ dental de l'hindi et un contraste vélaire vs uvulaire de la langue salish). Les plus jeunes bébés étaient tous capables de discriminer ces trois contrastes. Par contre, à l'âge de 8 à 10 mois, seuls certains des bébés conservaient la sensibilité pour les contrastes non natifs. À l'âge de 10 à 12 mois les bébés n'étaient plus capables de discriminer les deux contrastes des langues non familières; la 
sensibilité n'était maintenue que pour le contraste de la langue maternelle. Des résultats complémentaires ont été obtenus dans une étude longitudinale avec un nombre réduit de sujets, mais la tendance décrite ci-dessus a été confirmée. De plus, les bébés exposés au hindi et au salish comme langues maternelles ont montré la sensibilité à ces contrastes à l'âge de 12 mois. D'autres études ont pu mettre en évidence un déclin de la sensibilité à discriminer les consonnes vers la fin de la première année de vie, ce qui montre l'existence d'une réorganisation perceptive comme résultat de l'expérience linguistique (Best, 1994 ; Werker \& Lalonde, 1988).

Dans une étude différente, Best et al. (1988) ont testé des groupes différents de bébés (langue maternelle anglais) avec le contraste entre un click zoulou apical ou latéral. Dans ce cas, la discrimination a été observée à tous les âges étudiés, ainsi que pour les adultes anglophones chez qui ce type de contraste est, bien sûr, non distinctif (Best et al., 1988). Ceci montre que l'expérience linguistique n'est pas le seul facteur à considérer pour expliquer ces changements perceptuels dans la discrimination des sons. On peut penser que dans ce cas, les clicks n'ont pas donné lieu à un traitement catégoriel de type linguistique, s'appuyant sur une comparaison avec les sons de la langue maternelle. On peut en effet considérer que les clicks peuvent ne pas être assimilés à des sons consonantiques et comparés, à ce titre à l'inventaire phonologique de la langue maternelle, mais qu'ils sont plus probablement traités comme des «bruits».

Des processus de réorganisation perceptive ont également été identifiés pour les sons vocaliques, mais les études montrent qu'ils ont lieu de façon plus précoce au cours du développement. Une étude réalisée par Kuhl et al. (1992) a montré des effets spécifiques de la langue environnante sur la perception de voyelles dès l'âge de 6 mois (Kuhl et al., 1992). Deux groupes de bébés, américains et suédois, ont exécuté une tâche de discrimination de voyelles, impliquant, respectivement, la voyelle prototypique /i/ de l'anglais et la voyelle prototypique $/ y /$ du suédois comme stimuli de référence, à contraster avec différentes variantes autour de chacun de ces deux stimuli. Les résultats ont montré une capacité de discrimination réduite autour de la voyelle prototypique (/i/ pour les bébés américains et /y/ pour les bébés suédois), ce qui indiquerait que l'expérience linguistique joue déjà un rôle dans la formation des premières catégories vocaliques de la langue maternelle. Le modèle proposé par P. Kuhl, le Native Language Magnet Model (le Modèle de l'Aimant de Langue Native) explique entièrement ces biais perceptuels spécifiques de la langue qui reflètent le rôle de l'exposition linguistique (Kuhl, 1998 ; Kuhl, 2000). Une étude différente traite également de cette question du changement dans la perception de voyelles au cours de la première année de vie et, globalement, les résultats confirment un déclin 
dans les capacités de discrimination d'abord pour les voyelles (autour de l'âge de 6/8 mois), puis pour la plupart des consonnes qui n'appartiennent pas à la langue maternelle (Polka \& Werker, 1994).

Des études électrophysiologiques plus récentes ont confirmé ces changements spécifiques dans la perception des voyelles et des consonnes au cours de la première année de vie. L'étude des réponses électriques cérébrales (MMN, par exemple) pour des stimuli vocaliques spécifiques montre des réponses qui changent avec l'âge. Ces changements sont interprétés comme une modification du type de catégorisation opérée par les bébés. À un premier stade, vers 6 mois, les patrons de décharge électriques indiquent une catégorisation s'appuyant prioritairement sur des indices acoustiques, alors qu'à un deuxième stade, à la fin de la première année, la catégorisation reflète une analyse de type phonémique. La réorganisation des sites neurologiques a donc été interprétée comme une conséquence de la valeur phonémique que certains sons disponibles dans l'input acquièrent graduellement au cours du deuxième semestre de vie (Cheour et al., 1998). Une autre recherche a analysé les étapes impliquées dans la discrimination de phonèmes consonantiques. Elle a montré au moins deux étapes différentes qui correspondraient à une catégorisation de plus en plus raffinée reflétée par des patrons de réponse différenciés dans les lobes temporaux, là où les changements phonétiques sont détectés (Dehaene-Lambertz \& Dehaene, 1994).

La réorganisation perceptive orientée vers le développement progressif des catégories phonémiques chez le bilingue a été récemment étudiée dans notre laboratoire. Chez l'enfant bilingue, le résultat final de ces processus de réorganisation perceptive doit être compatible avec l'existence de deux systèmes qui correspondent aux deux langues présentes dans l'environnement. Si l'on considère que la formation des contrastes phonétiques spécifiques de la langue maternelle a lieu pendant le deuxième semestre de vie, d'abord pour les voyelles et ensuite pour les consonnes, et si l'on accepte que dans beaucoup de situations bilingues une différenciation entre les langues peut être atteinte avant l'âge de 6 mois, alors on pourrait envisager qu'il n'y ait pas de grandes différences entre les capacités de discrimination chez les bébés monolingues et les bilingues (si l'on considère comme facteur prioritaire que la différentiation précoce entre les langues facilite le processus de catégorisation relative aux deux systèmes de sons). Pourtant, des résultats obtenus dans notre laboratoire ont montré un patron de réponses spécifiques dans le groupe bilingue, différent de celui qu'on avait observé chez les enfants des familles monolingues (Bosch \& Sebastián-Gallés, 2003).

Des enfants de 4 et 8 mois, monolingues (espagnol ou catalan) et bilingues (espagnol et catalan) ont participé à une étude de discrimination avec une procédure de familiarisation-préférence sur un contraste vocalique 
qui n'existe qu'en catalan: /e/-/ع/ ([dedi] versus [dedi]). Comme prévu, à une étape très précoce, avant les 6 mois, les plus jeunes bébés étaient tout à fait capables de percevoir ce contraste, quelle que soit leur langue d'exposition. Cependant, à 8 mois, seuls les bébés des environnements monolingues catalans ont réussi à faire cette discrimination. Bien qu'un déclin dans la sensibilité ait été prévu pour le groupe espagnol monolingue, les résultats des bilingues étaient clairement imprévus, puisque le catalan est l'une des langues que ces bébés entendent régulièrement. Un groupe supplémentaire de bébés bilingues, âgés de 12 mois, a été testé afin d'analyser le décours temporel et la stabilité de ce patron particulier de réponses. Les résultats de cette étude supplémentaire à 12 mois ont montré que la discrimination était finalement atteinte, ce qui nous indiquerait que les enfants bilingues ont une conduite similaire à celle observée pour les monolingues mais retardée par rapport aux enfants monolingues qui sont quatre mois plus jeunes (Bosch \& Sebastián-Gallés, 2003). Ces résultats contredisent l'idée selon laquelle la simple exposition permettrait de maintenir la capacité de discrimination des contrastes. Les résultats suggèrent plutôt que l'on trouve, chez les bilingues, des processus de réorganisation perceptive spécifiques et que ces processus suivent un décours temporel différent que celui des monolingues puisqu'ils doivent faire face à la formation de deux systèmes phonologiques.

Une deuxième interprétation est également possible : le contraste spécifique du catalan que nous avions choisi présente des caractéristiques qui ont pu rendre la tâche plus complexe pour les enfants bilingues. En effet, ce contraste qui est distinctif en catalan est neutralisé pour un hispanophone où il n'existe qu'un seul degré d'aperture pour les voyelles moyennes. [e] et $[\varepsilon]$ sont donc, dans ce système, des réalisations allophoniques et non pas deux phonèmes différents comme pour les catalans. Ainsi, il est tout à fait possible d'interpréter le résultat à 8 mois comme une discrimination difficile à atteindre pour un bilingue espagnol-catalan. Cette interprétation invite à considérer que, dans un premier temps, les bébés bilingues interpréteraient ces deux voyelles moyennes comme des allophones, conformément à la phonologie de l'espagnol, et comme l'ont fait les bébés monolingues espagnols, puisqu'ils les distingueraient ensuite comme deux phonèmes différents.

Une troisième interprétation peut encore être envisagée du point de vue distributionnel. Les bébés exposés au catalan et à l'espagnol entendent dans leur expérience linguistique un nombre supérieur de voyelles du type /e/ que de voyelles de la catégorie /E/ (Bosch \& Sebastián-Gallés, 2003). Comme l'ont montré Maye et al. (2002), des enfants exposés à une distribution bimodale de paires de sons où est réalisé un contraste de VOT ( $v s$ enfants exposés à un contraste unimodal) établissent plus facilement le traitement catégoriel de paires d'occlusives voisées/non voisées. Par conséquent, 
les résultats peuvent s'interpréter comme un effet de fréquence distributionnelle. Les distributions respectives de /e/ et / $/ \varepsilon /$ auraient d'abord été interprétées comme une distribution unimodale où $/ \varepsilon /$ n'a pas été reconnu, les bébés ayant d'abord construit une seule catégorie autour de la voyelle la plus fréquente.

Si cette hypothèse est correcte, la discrimination ne peut pas être atteinte en un premier temps, et il faut attendre jusqu'à ce qu'un mécanisme déclenche la différenciation, par la reconnaissance d'une distribution bimodale de /e/ et / $/ \varepsilon /$ à laquelle les enfants n'ont pas été sensibles au départ. Un possible mécanisme pourrait être lié à l'incrément dans la connaissance lexicale qui se produit vers la fin de la première année de vie. Si cette interprétation est juste, nous devrions observer des processus similaires pour les autres contrastes de voyelles, surtout pour ceux qui sont peu fréquents ou qui ne peuvent pas s'assimiler à des contrastes dans l'autre langue quand les deux systèmes sont comparés. Les données plus récentes qui explorent les capacités de discrimination pour d'autres contrastes vocaliques dans notre laboratoire suggèrent que cette interprétation est plausible. Nous avons en effet observé que les bébés de 8 mois montrent une absence de discrimination pour des catégories contrastives même communes aux deux langues (par exemple, /o/-/u/), alors qu'à l'âge de 12 mois ils arrivent finalement à percevoir ces contrastes (Bosch \& Sebastián-Gallés, 2005). Le rôle de la quantité d'exposition à la langue qui est nécessaire pour construire ces catégories contrastives doit être considéré, mais aussi le nombre et la distribution des voyelles dans l'espace perceptif chez le bilingue qui est sans doute un facteur non négligeable pour interpréter ce délai dans la formation des catégories.

Une tendance similaire chez des enfants bilingues d'autres langues a été également observée dans un travail récent publié par Werker et collaborateurs (Burns et al., 2002). Dans une série d'expériences, ils ont examiné le décours du développement et la nature de représentations phonétiques relatives aux frontières catégorielles entre [b], [p] et [ph]. Dans la comparaison entre enfants monolingues anglais et bilingues anglais-français, à 12 mois, on observe une différence significative : les monolingues ont déjà placé une frontière catégorielle correspondant aux frontières existant dans leur langue, alors que les bilingues du même âge ne montrent pas encore de discrimination catégorielle correspondant aux catégories existant dans l'une ou l'autre de ces deux langues. Cependant, lorsqu'ils sont un peu plus âgés, entre 14/21 mois, les bébés bilingues montrent soit un comportement qui est identique à celui des monolingues dans une des deux langues, soit le comportement de systèmes monolingues parallèles. Ils établissent des discriminations catégorielles correspondant aux contrastes existant dans les deux langues auxquelles ils ont été exposés. 
Le parallélisme entre cette étude et les résultats obtenus dans notre laboratoire avec les bébés bilingues catalan-espagnol suggère que pendant une certaine période (à 8 mois dans l'étude de voyelles et à 10/12 mois dans l'étude de consonnes) ces bébés établissent un traitement catégoriel sur la base d'une des deux langues exclusivement, puis réorganisent ensuite leurs représentations phonologiques pour les ajuster aux catégories des deux langues. Pendant quelques mois le traitement catégoriel serait, temporairement, « en retard», par rapport à celui des monolingues.

\section{La connaissance des patrons phonotactiques spécifiques à la langue et leur rôle dans la segmentation de la parole continue}

Pendant la deuxième moitié de la première année de vie, les bébés acquièrent graduellement différents types de connaissances phonologiques qu'ils utilisent pour segmenter des mots possibles dans la parole continue. Un type particulier de connaissance concerne les séquences de segments qui se trouvent à l'intérieur des mots (et aussi entre les mots). Des recherches ont pu montrer que les bébés peuvent extraire les probabilités transitionnelles de segments à l'intérieur des mots et à travers les frontières de mots (Friederici \& Wessels, 1993 ; Jusczyk et al., 1994). Cette capacité leur permet de montrer une préférence pour des mots qui présentent des combinaisons de sons possibles dans leur langue (clusters consonantiques légaux en attaque en hollandais ou séquences phonotactiques fréquentes en anglais) et d'écarter des combinaisons impossibles ou peu fréquentes dans leur langue maternelle.

Il est possible de trouver et de segmenter des mots d'une façon fiable dans la parole continue dès l'âge de 10 mois d'abord à partir des indicateurs prosodiques, mais également grâce à l'utilisation d'informations de nature phonotactique et allophonique (Jusczyk et al., 1999; Myers et al., 1996).

Ces différentes sources d'information sont spécifiques à la langue (par exemple, l'accent est placé au début des mots en anglais, alors qu'en espagnol, il se trouve plus fréquemment sur l'avant-dernière syllabe). Cela signifie que l'enfant exposé à deux langues doit extraire deux types différents de régularités statistiques, lui permettant de segmenter respectivement les langues auxquelles il est exposé et d'identifier les mots possibles qui constitueront les vocabulaires correspondant aux deux langues cibles.

Ainsi, au-delà des traits prosodiques qui permettent d'abord une segmentation rudimentaire de la parole continue en unités lexicales (pour les bébés exposés à deux langues similaires du point de vue prosodique, cette propriété ne va pas être très utile pour séparer les deux lexiques), l'acquisition des connaissances phonotactiques spécifiques de chaque langue est 
considérée comme fondamentale pour réussir d'abord à segmenter et ensuite à construire les deux lexiques chez le bilingue.

Ce problème a été abordé dans une recherche qui compare des bébés monolingues et bilingues avec une procédure de préférence (HEAD-TURN PREFERENCE PROCEDURE) qui permet de tester la discrimination entre des listes de non-mots qui sont phonotactiquement possibles ou impossibles dans l'une des langues d'exposition des bilingues (par exemple, le catalan : SebastiánGallés \& Bosch, 2002). Les bébés de 10 mois étaient séparés en trois groupes : monolingue espagnol, monolingue catalan et bilingue espagnol-catalan (la moitié d'entre eux étant dominants du catalan et l'autre moitié dominants d'espagnol en fonction de la langue parlée par leur mère). Les stimuli étaient constitués par deux types de listes de non-mots de structure CVCC, dont les coda -CC étaient possibles ou impossibles en catalan et toujours impossibles en espagnol (l'espagnol n'a pas de codas complexes à la fin des mots, sauf exceptionnellement dans des mots empruntés à d'autres langues). Des résultats obtenus par Friederici \& Wessels (1993) et Jusczyk et al. (1994) avaient déjà montré la sensibilité des bébés de 9 mois à la phonotactique de leur langue maternelle, mais aucune indication n'existait sur l'acquisition de cette connaissance chez les bébés bilingues exposés à deux langues avec une structure prosodique similaire mais avec des patrons phonotactiques différents. Il est raisonnable de penser que les bébés exposés à deux langues dès la naissance ont quantitativement moins d'exposition à chacune d'elles que les bébés monolingues. Ainsi, on peut faire l'hypothèse que, pour un bilingue, cette sensibilité aux patrons phonotactiques va se développer plus lentement, étant donné que pour une même période de temps, leur exposition à une langue donnée est moindre. Dans ce cas, il est plausible de prédire que cette sensibilité va se montrer d'abord pour les patrons communs aux deux langues, même si les langues comportent des différences notables. Toutefois, on peut également supposer que la tendance inverse est possible : en focalisant l'attention sur les patrons différents entre les deux langues, les bébés pourraient exploiter une stratégie leur permettant d'augmenter la distance perceptuelle entre les langues et repérer très rapidement les contraintes phonotactiques qui caractérisent en propre chacune des deux langues. Ceci faciliterait la construction de deux systèmes bien différenciés. Deux hypothèses sont donc initialement possibles: l'une suggère un retard de traitement phonotactique chez le bilingue et l'autre au contraire plaide en faveur d'un rythme d'acquisition similaire entre bilingues et monolingues.

Par ailleurs, comme il y a deux groupes d'enfants bilingues dans cette étude, définis en fonction de leur langue «maternelle» dominante, une troisième possibilité doit être envisagée. La prédominance d'une langue dans 
l'exposition bilingue ferait que, seul le groupe catalan-dominant se comporterait de façon comparable au groupe monolingue catalan. Si c'était le cas, on mettrait en évidence un effet précoce de langue dominante chez le bilingue, bien qu'il ait été exposé simultanément à deux langues dès la naissance.

Les résultats obtenus plaident en faveur de cette dernière interprétation : les bébés monolingues espagnols n'ont montré aucune préférence entre les deux types de stimuli CVCC, les bébés monolingues catalans et bilingues dominants catalans ont montré un patron équivalent de préférence pour les stimuli «légaux », alors que les bébés bilingues dominants espagnols ont montré un patron ambigu, qui se situe globalement entre le patron des monolingues espagnols et celui des bilingues dominants catalans. Ces résultats, donc, sont plus en accord avec la notion d'une prédominance linguistique chez les bébés bilingues, conséquence d'une exposition plus longue à l'une des deux langues de l'environnement. Pour confirmer cette hypothèse, une recherche complémentaire est nécessaire, dans laquelle les bébés bilingues dominants espagnol montreraient aussi des réponses préférentielles pour des patrons phonotactiques présents uniquement en espagnol. Malheureusement, les propriétés phonotactiques de ces deux langues ne permettent pas de construire ce type d'étude et, par conséquent, cette hypothèse reste à confirmer.

\section{La représentation des mots dans le premier lexique des enfants bilingues}

Jusczyk \& Aslin (1995) ont montré que la capacité à segmenter des mots dans la parole continue était présente dès l'âge de 7 mois et demi, et que cette capacité reflète l'utilisation d'un degré important de détails phonétiques. Lorsque les mots à segmenter se différencient par leur consonne initiale (par exemple, cup _tup «tasse»), ils ne sont plus reconnus. Très récemment, cette capacité a été explorée chez les bébés bilingues d'environnements français/anglais (Polka et al., 2002). Les résultats indiquent qu'il n'y a pas de retard dans cette capacité lorsque le bilingue est comparé au monolingue (les bébés dans les deux groupes avaient 7 mois et demi). Le bilingue peut segmenter des mots bissyllabiques dans les deux langues d'exposition, y compris dans le cas de langues rythmiquement différentes et qui n'ont pas un patron accentuel semblable (FORT-FAIBLE en anglais et FAIBLEFORT en français). Si la tendance observée dans cette étude préliminaire est finalement confirmée, alors il sera nécessaire d'analyser si le bilingue développe des stratégies distinctes pour segmenter des mots dans chacune des langues d'exposition (c'est-à-dire, se comportant comme monolingue dans 
chacune des langues) ou bien si, au contraire, il utilise des stratégies de segmentation compatibles avec les deux langues. Cette dernière hypothèse consiste à dire que le bilingue développe des stratégies spécifiques probablement différentes de celles d'un enfant monolingue.

Quel genre d'information utilise le bébé pour reconnaître un mot? Quel niveau de détail phonétique est nécessaire pour la reconnaissance des mots ? La plupart des modèles de reconnaissance de mots chez l'adulte considèrent qu'il existe un accès très détaillé à une information phonétique précise. Pourtant, si l'on considère le nombre réduit d'unités lexicales chez le jeune enfant, ce niveau de détail peut ne pas être nécessaire au début, du moins jusqu'à ce qu'une certaine taille de vocabulaire soit atteinte.

Des études, développées principalement par J. Werker et collaborateurs (voir Werker \& Yeung, 2005, pour une synthèse sur ces études) montrent une continuité entre la formation des catégories phonétiques prélexicales (développées pendant le deuxième semestre de vie) et l'usage de ces catégories dans les représentations des mots (une fois que l'enfant a commencé à construire son vocabulaire réceptif). D'autres recherches sur la reconnaissance de mots familiers à l'âge de 18 à 23 mois ont suggéré qu'à cet âge les représentations sont assez bien spécifiées phonétiquement (Swingley \& Aslin, 2002). Cependant, d'autres résultats controversés ont montré des limitations dans l'usage des sensibilités spécifiques de la langue pour une tâche d'apprentissage de mots (Stager \& Werker, 1997). En d'autres termes, les bébés de 14 mois n'étaient pas capables de distinguer la paire minimale formée par les non-mots [bih]-[dih] appliqués à deux objets différents, alors que ce contraste pouvait être perçu et différencié lorsque les stimuli n'étaient pas associés à des objets. Des expériences additionnelles ont montré que cette incapacité n'était plus présente à l'âge de 17/20 mois. Une explication possible pour ce déficit temporaire est de le mettre en relation avec un excès de demandes computationnelles relatives à la situation de test.

Le traitement sémantique qui est nécessaire pour attribuer au contraste [bih]-[dih] le statut de paire minimale constituerait une «surcharge cognitive » rendant la tâche impossible pour un bébé de 14 mois. Au fur et à mesure que l'enfant acquiert la possibilité de faire les associations motsobjets, il peut appliquer ses connaissances des catégories contrastives dans la représentation des mots. Selon l'hypothèse proposée par Werker \& Fennell (2004), seuls les enfants plus âgés, ou avec un vocabulaire plus large, seraient capables d'utiliser le détail phonétique dans la tâche d'apprentissage de nouveaux mots.

Le comportement des enfants bilingues dans ce type de tâche d'apprentissage de mots a également été étudié par le groupe canadien de J. Werker. 
D'après leur hypothèse, il existe une limitation de ressources attentionnelles qui interfèrerait temporairement avec la capacité à apprendre des paires minimales. De ce point de vue, la population bilingue est spécialement intéressante, si l'on considère en particulier l'augmentation des demandes attentionnelles que suppose l'acquisition simultanée de deux systèmes lexicaux. Les résultats de leurs recherches ont indiqué que les enfants bilingues montrent non seulement une difficulté similaire à celle des monolingues à l'âge de 14 mois, mais qu'ils continuent à montrer le même résultat à 17 mois, moment auquel le groupe des monolingues est déjà capable d'apprendre correctement les nouvelles paires minimales (Fennell \& Werker, 2000). Ce résultat semble indiquer que, bien qu'ils aient été exposés à deux systèmes linguistiques différents, les enfants bilingues n'utilisent pas plus de détail phonétique quand ils apprennent des étiquettes phonétiquement similaires pour des nouveaux objets. L'accès au détail phonologique dans la représentation des mots semble être atteint plus tard chez le bilingue que chez le monolingue, au moins en ce qui concerne cette tâche d'apprentissage de mots. Des nouvelles recherches, avec une approche méthodologique différente, sont nécessaires pour permettre de confirmer cette interprétation.

Une autre manière d'explorer les caractéristiques du format de représentation des mots dans le processus de développement lexical consiste à analyser la réaction des bébés face à des modifications de la forme phonétique de mots familiers. Des résultats en anglais montrent une sensibilité importante à la forme phonétique des mots connus chez l'enfant monolingue, indépendamment de l'âge ou du niveau de vocabulaire des enfants (Swingley \& Aslin, 2000 ; Bailey \& Plunkett, 2002). Les données récemment obtenues dans notre laboratoire sur la reconnaissance de mots bien et mal prononcés ont montré comment des enfants bilingues simultanés catalan-espagnol, d'âges compris entre 18 et 24 mois montrent des difficultés pour codifier le détail phonologique. Notre étude faisait usage de mots familiers et testait le contraste vocalique exclusif du catalan, /e/-/ع/. Les enfants n'ont pas été capables de réagir devant des mots mal prononcés, créés par la substitution de ces voyelles, tandis qu'au même âge, les enfants des familles monolingues (exclusivement catalan) ont réussi à faire cette même distinction. De plus, les enfants bilingues ont réagi a un changement de voyelle uniquement sur un son vocalique commun aux deux langues. Par conséquent, il est raisonnable d'avancer que ces enfants codifient ce type de détail phonologique, mais avec des restrictions liées à la relation entre les contrastes vocaliques des deux systèmes phonologiques (Ramon-Casas et al., 2005).

Comment peut-on expliquer ces résultats obtenus chez le bilingue catalan-espagnol dans une tâche de reconnaissance de mots familiers ? On 
savait déjà que ces bébés sont capables de percevoir ce contraste vocalique dès l'âge de 12 mois, bien qu'ils soient en retard par rapport aux bébés des familles monolingues. Ces derniers réussissent à faire la discrimination à 8 mois, et les bilingues à 12 mois (Bosch \& Sebastián-Gallés, 2003). Quelques mois plus tard, au moment d'utiliser ces connaissances phonétiques dans la reconnaissance des mots, ils semblent à nouveau être en retard par rapport aux enfants monolingues. Différents types d'explications sont possibles et le rôle de certains facteurs, soit relatifs au décours du développement, soit strictement phonologiques, doit être analysé. Si les études précédentes dans notre laboratoire ont pu montrer des patrons de réponse spécifiques de la population bilingue et des processus de réorganisation perceptive différents entre bilingues et monolingues, il est possible que l'on ait identifié un nouveau trait distinctif entre ces deux populations qui concernerait le format initial de représentation des mots. On peut faire l'hypothèse qu'une nouvelle période de réorganisation perceptive a lieu au moment où les sensibilités phonétiques sont appliquée à la représentation des premiers mots des deux lexiques (Bosch \& Ramon-Casas, 2005). Nous discutons ci-dessous les possibles interprétations de ce phénomène.

La différence observée peut dériver des caractéristiques quantitatives et qualitatives propres à l'exposition linguistique. Apparemment, la quantité d'exposition à la langue peut être une variable décisive au moment de prédire la réussite d'une tâche d'apprentissage de mots (Fennell et al., 2000). Ce même facteur peut être déterminant pour une tâche de reconnaissance de mots, bien qu'il s'agisse d'une tâche qui exige probablement moins de ressources attentionnelles. S'il s'agit simplement d'une question quantitative, alors on devrait observer une augmentation, avec l'âge, de la sensibilité au détail phonétique et à la réalisation erronée d'un mot, chez les enfants monolingues et bilingues entre 18 et 24 mois.

Mais il y a aussi un autre élément à considérer dans l'exposition bilingue : l'enfant est possiblement exposé à des prononciations plus variables d'un même mot, et à des réalisations qui pourraient même inclure des versions «mal prononcés », comme celles qui sont utilisées dans le test. Pour cette raison, le bilingue serait plus tolérant aux versions mal prononcées des mots présentés dans l'étude effectuée et, dans ce cas-là, il ne réagirait pas à des modifications de réalisation auxquelles le monolingue serait sensible plus tôt.

Un autre élément à considérer est celui qui concerne le type de voyelles que l'on contraste et que l'on utilise pour construire les mots mal prononcés. Il ne faut pas oublier que le contraste vocalique testé est un contraste spécialement problématique (non commun aux deux langues, et 
surtout où l'une des langues neutralise l'autre) et, en outre, très proche dans l'espace acoustique. Les bilingues grouperaient dans la même catégorie ces deux exemplaires de voyelles, au moins dans une première étape du développement lexical. Le changement, dans ce cas, ne serait possible que plus tardivement, lorsque les deux lexiques auraient acquis un niveau de spécification phonologique plus complet.

À nouveau, un test à un âge supérieur devient nécessaire pour analyser l'éventuelle utilisation de ce contraste «tardif », ce qui pourrait indiquer que la sensibilité diffère selon le contraste vocalique utilisé.

Des résultats précédents obtenus dans notre laboratoire ont mis en évidence que tous les contrastes ne sont pas également disponibles, même chez les bilingues équilibrés, qu'il s'agisse d'enfants (Bosch \& SebastiánGallés, 2003) ou d'adultes (Sebastián-Gallés \& Soto-Faraco, 1999). Ceci invite à considérer que l'hypothèse d'une langue dominante est pertinente même chez le bilingue. Bien que dans notre étude cette hypothèse d'une dominance de la langue «maternelle » (c'est-à-dire, de la langue qui a une présence prédominante dans l'environnement), ne puisse pas constituer l'explication univoque des résultats des bilingues, il est vrai qu'un test avec des enfants plus âgés pourrait mettre en évidence l'existence d'une langue prioritaire, sur laquelle les contrastes auraient un statut semblable à celui qu'on observe chez le monolingue.

Le rôle de ces différents facteurs est en train d'être analysé dans notre laboratoire. Pour cela, nous réalisons de nouvelles séries d'expériences auxquelles participent des groupes d'enfants bilingues et monolingues âgés de 3 à 4 ans, ainsi que des enfants plus jeunes testés avec des stimuli linguistiques différents.

Des résultats tout à fait préliminaires semblent indiquer que le contraste catalan /e/-/E/ est finalement utilisé pour identifier les mots mal prononcés, bien que ce ne soit pas une tendance présente chez tous les enfants. La langue dominante dans l'entourage linguistique semble jouer un rôle dans le traitement de ce contraste exclusif d'une des deux langues auxquelles les enfants sont exposés. Si cette tendance se confirme, les résultats suggèreraient, chez les bilingues, des processus différents pour catégoriser les contrastes «conflictuels », du fait des propriétés spécifiques des sons (acoustiques et relatives à la fréquence d'occurrence dans les deux langues) ou du fait de leur réalisation par l'entourage (présence de « mauvaises prononciations »).

Une explication alternative et récente, qui a été proposée par Nespor et al. (2003) et testée expérimentalement (Nazzi, sous presse), à propos du rôle distinct des sons vocaliques et consonantiques dans le traitement de la parole, est difficilement compatible avec les données obtenues chez les 
bilingues. L'étude réalisée avec des enfants français pour explorer la spécificité des voyelles dans les premières représentations lexicales montre qu'à l'âge de 20 mois, dans une tâche de catégorisation de nouveaux mots, les enfants ont plus de problèmes pour apprendre deux mots qui sont uniquement distingués par une voyelle, que si les mots se différencient par une consonne. Ces premiers résultats ainsi que le fait que la majorité des études sur la reconnaissance de mots ont utilisé des contrastes consonantiques, invitent à s'interroger sur les résultats que nous avons obtenus.

Les effets qui ont été identifiés dans notre recherche sur la reconnaissance des mots chez les bilingues catalan-espagnol n'auraient peut-être pas été observés aussi clairement si l'on avait utilisé un contraste consonantique, même s'il est exclusif d'une des deux langues. On peut se demander en particulier si le décalage temporel, que nous avons systématiquement mis en évidence entre les monolingues et les bilingues, serait observable sur la base d'expériences testant des contrastes consonantiques.

Toutes ces possibilités restent ouvertes et attendent que de nouvelles expériences, dont quelques-unes sont en cours de réalisation, offrent des réponses qui nous permettent d'avancer dans le débat actuel sur le format de représentation des mots dans les premières étapes de l'acquisition du lexique.

\section{NOTE}

1. James S. McDonnell Foundation (Bridging Brain, Mind and Behavior Program); bourse au projet SEJ2004-06429/PSIC du Ministerio de Educación y Ciencia (Espagne) et aide à la recherche 2001SGR00034 aux groupes consolidés de l'Université de Barcelone. Nous remercions aussi F. Xavier Alario pour les commentaires et la révision du texte en français de ce manuscrit. 


\section{RÉFÉRENCES BIBLIOGRAPHIQUES}

Abercrombie, David (1967). Elements of general phonetics. Edinburgh : Edinburgh University Press.

Bailey, Todd M. ; Plunkett, Kim (2002). Phonological specificity in early words. Cognitive Development, 17 : 1265-1282.

BAHRick, Lorraine E. ; Pickens, Jeffrey N. (1988). Classification of bimodal English and Spanish language passages by infants. Infant Behaviour and Development, 11 : 277-296.

BEST, Catherine T. (1994). The emergence of native-language phonological influence in infants: A perceptual assimilation model. Dans Goodman, J. C. \& Nusbaum, H. C. (eds.). The development of speech perception: The transition from speech sounds to spoken words, 167-224. Cambridge, MA : MIT Press.

Best, Catherine T. ; McRoberts, Gerald W. ; Sithole, Nomathemba M. (1988). The phonological basis of perceptual loss for non-native contrasts : maintenance of discrimination among Zulu clicks by English-speaking adults and infants. Journal of Experimental Psychology: Human Perception and Performance, $14: 345-360$.

Bosch, Laura (2000). The impact of bilingual exposure on early speech perception : The discrimination of vowel contrasts. Paper presented at the workshop: Development and interaction of linguistic and non-linguistic cognition in infants. Berlin.

Bosch, Laura ; Cortes, Carolina ; Sebastián-Gallés, Núria (2001). El reconocimiento temprano de la lengua materna : un estudio basado en la voz masculina. Infancia y Aprendizaje, 24 (2) : 197-213.

Bosch, Laura ; RAmon-CASAS, Marta (2005). Continuity or discontinuities in developing bilinguals' representation of speech ? Paper presented at the $5^{\text {th }}$ International Symposium on Bilingualism. Barcelona.

Bosch, Laura ; Sebastián-Gallés, Núria (1997). Native-language recognition abilities in four-month-old infants from monolingual and bilingual environments. Cognition, 65 : 33-69.

Bosch, Laura ; Sebastián-Gallés, Núria (2000). Exploring four-month-old infants' abilities to discriminate languages from the same rhythmic class. Paper presented at the International Conference on Infant Studies, Brighton, UK.

Bosch, Laura ; SEBAStián-Gallés, Núria (2001a). Evidence of early language discrimination abilities in infants from bilingual environments. Infancy, 2 : 29-49.

Bosch, Laura; Sebastián-Gallés, Núria (2001b). Early language differentiation in bilingual infants. Dans Cenoz, J. \& Genesee, F. (eds.). Trends in bilingual acquisition, 71-93. Amsterdam : John Benjamins Publ. Co.

Bosch, Laura; Sebastián-Gallés, Núria (2003). Simultaneous bilingualism and the perception of a language specific vowel contrast in the first year of life. Language and Speech, $46: 217-243$.

Bosch, Laura; Sebastián-Gallés, Núria (2004). Distributional properties of vowel sounds in fluent speech as a cue to discriminate languages. Poster presented at the XIV th International Conference on Infant Studies. Chicago. 
Bosch, Laura ; Sebastián-Gallés, Núria (2005). Developmental changes in the discrimination of vowel contrasts in bilingual infants. Dans Cohen, McAlister, Rolstad, \& MacSwan (eds.), ISB4 : Proceedings of the 4th International Symposium on Bilingualism. Sommerville, MA : Cascadilla Press.

Burns, Tracey C. ; Werker, Janet F. ; McVIE, Karen (2002). Development of phonetic categories in infants raised in bilingual and monolingual environments. Paper presented at the Boston University Conference on Language Development. Boston, Mass.

Cheour, Marie ; Ceponiene, Rita ; Lehtokoski, Anne ; Luuk, Aavo ; Allik, Jüri ; AlHo, Kimmo ; NÄÄTÄNEn, Risto (1998). Development of language-specific phoneme representations in the infant brain. Nature Neuroscience, 1 : 351353.

Christophe, Anne ; Morton, John (1998). Is Dutch native English ? Linguistic analysis by two-month-olds. Developmental Science, 1 : p. 215-219.

Dehaene-Lambertz, Ghislaine ; Dehaene, Stanislas (1994). Speed and cerebral correlates of syllable discrimination in infants. Nature, $370: 29$ 2-295.

Dehaene-Lambertz, Ghislaine ; Houston, Derek (1998). Faster orientation latencies toward native language in two month old infants. Language and Speech, $41(1): 21-43$.

Fennell, Christopher T. ; Werker, Janet F. (2000). Does bilingual exposure affect infants' use of phonetic detail in a word learning task? Poster presented at the ICIS 2000 meeting. Brighton.

Friederici, Angela D. ; Wessels, Jeanine M. I. (1993). Phonotactic knowledge and its use in infant speech perception. Perception and Psychophysics, 54: 287-295.

Guasti, Maria Teresa ; Nespor, Marina; Christophe, Ann.; Van Ooyen, Brit (2001). Pre-lexical setting of the head-complement parameter through prosody. Dans Weissenborn, J. \& Hoehle, B. (eds.), How to get into language : Approaches to bootstrapping early language development, 231-248. New York : Benjamin.

JuSCZYK, Peter W. ; AsLin, Richard N. (1995). Infants' detection of sound patterns of words in fluent speech. Cognitive Psychology, 29 : 1-23.

JusczyK, Peter W. ; Hohne, Elisabeth A. ; Bauman, Angela (1999). Infants's sensitivity to allophonic cues for word segmentation. Perception and Psychophysics, 61 (8) : 1465-1476.

JusczyK, Peter W. ; Luce, Paul A. ; Charles-Luce, Jan (1994). Infants' sensitivity to phonotactic patterns in the native language. Journal of Memory and Language, 33 (5) : 630-645.

Kunl, Patricia (1998). The development of speech and language. Dans Carew, T. J., Menzel, R. \& Shatz, C. J. (eds.), Mechanistic relationships between development and learning, 53-73. New York: Wiley.

Kunl, Patricia (2000). Language, mind, and brain: Experience alters perception. Dans Gazzaniga, M. S. (ed.), The new cognitive neurosciences $\left(2^{\text {nd }}\right.$ ed.), 99115. Cambridge, MA : MIT Press. 
Kuhl, Patricia ; Williams, Karen A. ; Lacerda, Francisco. ; Stevens, Kenneth N. ; LindBLOM, Björn (1992). Linguistic experience alters phonetic perception in infants by 6 months of age. Science, $255:$ 606-608.

MAye, Jessica ; Werker, Janet F. ; Gerken, LouAnn (2002). Infant sensitivity to distributional information can affect phonetic discrimination. Cognition, 82 : B101-B111.

Mehler, Jacques ; Cristophe, Anne (1994). Language in the infant's mind. Philosophical transactions of the Royal Society of London, B (346) : 13-20.

Mehler, Jaques ; Dupoux, Emmanuel ; Nazzi, Thierry ; Dehaene-Lambertz, Ghislaine (1996). Coping with linguistic diversity: the infant's viewpoint. Dans Morgan J. L. \& Demuth, K. (eds.), Signal to syntax, 101-116. Mahwah, NJ : Lawrence Erlbaum Associates.

Mehler, Jaques ; JusczyK, Peter W. ; Lambertz, Ghislaine ; Halsted, Nilofar ; BerTOCini, Josiane; Amiel-Tison, Claudine (1988). A precursor of language acquisition in young infants. Cognition, 29:143-178.

Moon, Christine ; COOPER, Robin P. ; FIFER, William P. (1993). Two-day-olds prefer their native language. Infant Behaviour and Development, 16 (4) : 495-500.

Myers, James ; Jusczyk, Peter W. ; Kemler-Nelson, Deborah G. ; Charles Luce, Jan ; Woodward, Amanda ; Hirsh-PASEK, Kathryn (1996). Infants' sensitivity to word boundaries in fluent speech. Journal of Child Language, 23 : 1-30.

NAZZI, Thierry ; Bertocini, Josiane ; Mehler, Jaques (1998). Language discrimination by newborns : towards an understanding of the role of rhythm. Journal of Experimental Psychology : Human Perception and Performance, 24 : 756766.

NAZZI, Thierry ; JusczyK, Peter W. ; Johnson Elisabeth K. (2000). Language discrimination by English-learning 5-month-olds : Effects of rhythm and familiarity. Journal of Memory and Language, 43 : 1-19.

NAZZI, Thierry (2004). Use of phonetic specificity during the acquisition of new words : differences between consonants and vowels. Cognition, 20 : 1-18.

Nespor, Marina ; PeÑA, Marcela (2003). On the different roles of vowels and consonants in speech processing and language acquisition. Lingue e Linguaggio, ii : 221-247.

Polka, Linda; Sundara, Megha; Blue, Stephanie (2002). The impact of language experience on word recognition. Paper presented at the $143^{\text {rd }}$ meeting of the Acoustic Society of America. Pittsburgh.

PolkA, Linda; Werker, Janet F. (1994). Developmental changes in perception of non-native vowel contrasts. Journal of Experimental Psychology: Human Perception and Performance, 20 : 421-435.

Ramon-Casas, Marta ; Bosch, Laura ; Swingley, Daniel \& Sebastián-Gallés, Núria (2005). Early word recognition in bilinguals : differential sensitivity to vowel mispronunciations in known words. Paper presented at the $X^{\text {th }}$ International Congress for the Study of Child Language. Berlin, July 25-29, 2005. 
Ramus, Frank ; Hauser, Marc D. ; Miller, Cory ; Morris, Dylan ; Mehler, Jaques (2000). Language discrimination by human newborns and by cotton-top tamarin monkeys. Science, $288: 349-351$.

Ramus, Frank; Nespor, Marina; Mehler, Jaques (1999). Correlates of linguistic rhythm in the speech signal. Cognition, $73: 265-292$.

Sebastián-Gallés, Núria ; Bosch, Laura (2002). The building of phonotactic knowledge in bilinguals : The role of early exposure. Journal of Experimental Psychology: Human Perception and Performance, 28 : 974-989.

Stager, Christine L.; Werker, Janet F. (1997). Infants listen for more phonetic detail in speech perception than in word-learning tasks. Nature, 388 : 381382.

Swingley, Daniel ; Aslin, Richard N. (2000). Spoken word recognition and lexical representation in very young children. Cognition, $76: 147-166$.

Swingley, Daniel ; Aslin, Richard N. (2002). Lexical neighborhoods and the wordform representations of 14-month-olds. Psychological Science, 13 : 480- 484.

Toro, Juan Manuel; Trobalon, Josep Batista; Sebastián-Gallés, Núria (2005). Effects of Backward Speech and Speaker Variability in Language Discrimination by Rats. Journal of Experimental Psychology : Animal Behavior Processes, 30 (1) : 95-100

Werker, Janet F. ; Fennell, Christopher T. (2004). Listening to sounds versus listening to words : Early steps in word learning. Dans Hall, D. G. \& Waxman, S. (eds.), Weaving a lexicon. Cambridge, Mass. : MIT Press.

Werker, Janet F. ; LAlonde, Chris E. (1988). Cross-language speech perception : Initial capabilities and developmental change. Developmental Psychology, 24 : 672-683.

Werker, Janet F. ; TeEs, Richard C. (1984). Cross-language speech perception : Evidence for perceptual reorganization during the first year of life. Infant Behavior and Development, $7: 49-63$.

Werker, Janet F. ; Yeung, H. Henny (2005). Infant speech perception bootstraps word learning. Trends in Cognitive Sciences, 9 : 519-527. 


\begin{abstract}
This paper analyses the building of contrastive sound categories in bilingual's early phonological development. Research focused on early capacity to differentiate between the languages in the environment, sound discrimination in the prelexical infant, the development of phonotactic sensitivities and the continuity between these early abilities and their use in familiar word recognition in the second year of life, are successively revised. The comparison between two different language acquisition situations, monolingual and bilingual, offers the possibility to analyse the role of linguistic exposure in early phonological organisation. Research results indicate that the ability to distinguish between the languages is at place by 4 months of age, even for languages with similar rhythmic properties. Perceptual reorganization processes that take place by the end of the first year of life and that show the initial building of contrastive sound categories follow a different time-course in bilingual acquisition, when compared with monolingual data. Growing knowledge of phonotactic patterns even suggests the existence of language dominance, favouring the most frequent language in their environment. Finally, work dealing with familiar word recognition and format of representation by age two suggests a possible delay concerning the representation of a vowel contrast that belongs to one of the languages in the environment. Converging evidence from all the work revised supports the specificity of early phonological development in bilingual acquisition as a consequence of particular adaptations that take place in order to cope with the richer and more complex nature of their linguistic input.
\end{abstract}

\title{
KEYWORDS
}

Bilingual acquisition, speech perception, contrastive categories, word recognition, early phonology. 